\title{
Theoretical Outage Analysis of Nonlinear OFDM Systems with AF Relays
}

\author{
C. Alexandre R. Fernandes and Éder J. P. Farias
}

\begin{abstract}
In this work, a performance analysis of the outage probability of a cooperative diversity OFDM system is presented, considering the distortions introduced by nonlinear power amplifiers (PAs) at the source and relay nodes. A closed form expression for the instantaneous SNR is developed and analytical expressions for the outage probability are derived, considering an amplify-and-forward (AF) relay and Rayleigh fading. Both the maximum ratio combing (MRC) and selection combining (SC) methods are considered. The derived expressions show how some PA parameters affect the outage probability and diversity order. Computer simulation results illustrate and confirm the proposed theoretical expressions under different scenarios.
\end{abstract}

Index Terms-OFDM, cooperative communication, power amplifiers, nonlinear distortion, outage probability.

\section{INTRODUCTION}

Cooperative diversity has become a promising technology for the future wireless communication networks due to increased capacity, spatial diversity gains and enhanced coverage, without the need of multiple antennas [1]. On the other hand, orthogonal frequency division multiplexing (OFDM) is a well-known solution to wireless communications that has been adopted by several standards, such as IEEE 802.11a, IEEE 802.16, DVB and 3GPP Long Term Evolution (LTE) [2]. One of the main drawbacks of OFDM is the high peak-to-average power ratio (PAPR) of the transmitted signals [3], which may introduce nonlinear distortions in the received signals, due to the presence of nonlinear power amplifiers (PAs).

In this work, a theoretical performance analysis of the outage probability of a cooperative diversity OFDM system is presented, taking the nonlinear distortions introduced by nonlinear PAs into account. Specifically, an exact closed form expression for the instantaneous SNR is developed and approximated closed-form expressions for the outage probability are derived, considering an amplify-and-forward (AF) relay. In our analysis, we assume frequency-selective Rayleigh fading, with both the source and relay nodes having a nonlinear PA. Moreover, both maximum ratio combing (MRC) and selection combining (SC) methods are used to combine the signals received from the direct link with those received from the relay link.

C. Alexandre R. Fernandes is with the Department of Computer Engineering, Federal University of Ceará, R. Estanislau Frota s/n, 62010-560, Sobral/CE, Brazil. e-mail:alexandrefernandes@ufc.br. C. A. R. Fernandes is partially supported by $\mathrm{CNPq} /$ Brazil.

Éder J. P. Farias is with the Instituto Superior de Teologia Aplicada, R. Coronel Antônio Rodrigues Magalhães, 359, 62050-100, Sobral/CE, Brazil.. e-mail: eder@inta.edu.br.

Digital Object Identifier: 10.14209/jcis.2015.13.
It is shown that the outage probability strongly depends on the signal-to-noise ratio (SNR) threshold and on the SNR associated with the nonlinear PAs. In fact, several expressions for the outage probability can be derived, depending on the value of these parameters, showing how the PA nonlinearities affect the outage probability and the diversity order of the system, for both the MRC and SC methods. Computer simulations confirm the validity of the proposed theoretical expressions.

Some previous works have presented performance studies of cooperative communication systems with nonlinear distortions. In [4], [5], symbol error analysis is performed for AF cooperative systems, assuming that both the base station and the relay have nonlinear PAs. However, in those papers, it is assumed that the source-relay channel is time-invariant and that there is no direct path between the source and the destination. In addition, no outage analysis is carried out. In [6], [7], outage and symbol error analysis of an AF cooperative nonlinear OFDM system is developed. However, they assume that the PA of the source is linear. An outage analysis of an AF cooperative clipped OFDM transmission is presented in [8]. However, it is assumed that the PA of the relay is linear and that there is no direct path between the source and the destination.

The rest of the paper is organized as follows. In Section II, the system model is described. In Section III, the exact instantaneous SNR of the considered system is presented. The outage analysis of the MRC and SC receivers are derived in Sections V and IV, respectively. In Section VII, computer simulations that validate the proposed outage analysis are presented. Conclusions and perspectives are drawn in Section VIII.

\section{System MODEL}

Let us consider a dual-hop OFDM cooperative communication system composed of three half-duplex nodes: one source $(\mathrm{S})$, one relay $(\mathrm{R})$ and one destination (D). TDMA is used to provide orthogonal access for the three nodes. In the first time slot, node $\mathrm{S}$ transmits the information to nodes $\mathrm{R}$ and $\mathrm{D}$, and, in the second time slot, the node $\mathrm{R}$ retransmits the information to node D using the AF protocol. It is assumed that all the nodes have a single antenna and that they are synchronized at symbol level. Moreover, all the wireless links have frequency selective Rayleigh fading and the nodes $\mathrm{S}$ and $\mathrm{R}$ are equipped with a PA modeled by the nonlinear memoryless functions $f^{S}(\cdot)$ and $f^{R}(\cdot)$, respectively.

As a transmitted OFDM signal in the time domain can be modeled as a complex Gaussian random process, using the extension of the Bussgang's theorem, the signal transmitted 
by the source at the $n$th subcarrier in the frequency-domain can be written as [3]

$$
u_{n}^{(S)}=\alpha^{(S)} s_{n}+d_{n}^{(S)}
$$

where $\alpha^{(S)}$ is a scalar gain, $d_{n}^{(S)}$ is a nonlinear distortion (NLD) and $s_{n}$ is the transmitted signal, which is assumed to be independent and identically distributed (i.i.d.). The signal $d_{n}^{(S)}$ is a white complex Gaussian process uncorrelated with $s_{n}$ [3], with variance denoted by $\sigma_{d^{(S)}}^{2}$. For some functions $f^{S}(\cdot)$, there are analytical expressions for the gain $\alpha^{(S)}$ and for the NLD variance $\sigma_{d^{(S)}}^{2}[3]$.

In the frequency-domain, the signals received by the nodes $\mathrm{D}$ and $\mathrm{R}$ at the $n$th subcarrier are respectively given by

$$
\begin{aligned}
x_{n}^{(S D)} & =h_{n}^{(S D)} u_{n}^{(S)}+\eta_{n}^{(S D)} \\
& =h_{n}^{(S D)} \alpha^{(S)} s_{n}+h_{n}^{(S D)} d_{n}^{(S)}+\eta_{n}^{(S D)},
\end{aligned}
$$

and

$$
x_{n}^{(S R)}=h_{n}^{(S R)} \alpha^{(S)} s_{n}+h_{n}^{(S R)} d_{n}^{(S)}+\eta_{n}^{(S R)},
$$

where $h_{n}^{(S D)}$ and $h_{n}^{(S R)}$ are respectively the channel frequency responses of the SD and SR links at the $n$th subcarrier, and $\eta_{n}^{(S D)}$ and $\eta_{n}^{(S R)}$ are the corresponding additive white Gaussian noise (AWGN) components. All the AWGN terms are assumed to have the same variance, denoted by $\sigma_{\eta}^{2}$.

At node $\mathrm{R}$, the signal $x_{n}^{(S R)}$ is multiplied by a variable gain given by

$$
g_{n}=\frac{\sqrt{P_{r}}}{\sqrt{\left|h_{n}^{(S R)}\right|^{2}\left|\alpha^{(S)}\right|^{2} P_{s}+\left|h_{n}^{(S R)}\right|^{2} \sigma_{d^{(S)}}^{2}+\sigma_{\eta}^{2}}},
$$

where $E\{\cdot\}$ is the expectation operator, $P_{s}$ and $P_{r}$ are the transmission powers of the source and relay, respectively. Note that $\left[\left|h_{n}^{(S R)}\right|^{2}\left|\alpha^{(S)}\right|^{2} P_{s}+\left|h_{n}^{(S R)}\right|^{2} \sigma_{d^{(S)}}^{2}+\sigma_{\eta}^{2}\right]$ corresponds to the instantaneous power of the signal $x_{n}^{(S R)}$.

Similarly, at the second time slot, the signal transmitted by the relay and the signal received by the destination at the $n$th subcarrier can be written respectively as

$$
u_{n}^{(R)}=\alpha^{(R)} g_{n} x_{n}^{(S R)}+d_{n}^{(R)}
$$

and

$$
\begin{aligned}
x_{n}^{(S R D)} & =h_{n}^{(R D)} u_{n}^{(R)}+\eta_{n}^{(R D)} \\
& =h_{n}^{(R D)} h_{n}^{(S R)} \alpha^{(S)} \alpha^{(R)} g_{n} s_{n}+h_{n}^{(R D)} d_{n}^{(R)}+\eta_{n}^{(R D)} \\
& +h_{n}^{(S R)} h_{n}^{(R D)} \alpha^{(R)} g_{n} d_{n}^{(S)}+h_{n}^{(R D)} \alpha^{(R)} g_{n} \eta_{n}^{(S R)}, \quad(6)
\end{aligned}
$$

where $\alpha^{(R)}$ is a constant gain, $d_{n}^{(R)}$ is the NLD introduced by the relay, uncorrelated with $g_{n} x_{n}^{(S D)}, h_{n}^{(R D)}$ is the channel response of the RD link at the $n$th subcarrier and $\eta_{n}^{(R D)}$ is the corresponding AWGN component.

Equations (2) and (6) can then be expressed respectively as

$$
x_{n}^{(S R D)}=h_{1 n} s_{n}+\nu_{1 n}
$$

and

$$
x_{n}^{(S D)}=h_{2 n} s_{n}+\nu_{2 n},
$$

with

$$
\begin{gathered}
h_{1 n}=h_{n}^{(R D)} h_{n}^{(S R)} \alpha^{(S)} \alpha^{(R)} g_{n}, \\
h_{2 n}=h_{n}^{(S D)} \alpha^{(S)}, \\
\nu_{1 n}=h_{n}^{(S R)} h_{n}^{(R D)} \alpha^{(R)} g_{n} d_{n}^{(S)}+h_{n}^{(R D)} \alpha^{(R)} g_{n} \eta_{n}^{(S R)} \\
+h_{n}^{(R D)} d_{n}^{(R)}+\eta_{n}^{(R D)}, \\
\nu_{2 n}=h_{n}^{(S D)} d_{n}^{(S)}+\eta_{n}^{(S D)} .
\end{gathered}
$$

\section{INSTANTANEOUS SNR WITH MRC}

In this section, an expression for the exact instantaneous SNR of the system considered in Section II is presented, for a MRC receiver. Note that, due to the presence of the NLD term $d_{n}^{(S)}$, the noise components $\nu_{1 n}$ and $\nu_{2 n}$, defined in Section II, are correlated, which means that a pre-whitening step is required before the application of the MRC combiner [9]. The pre-whitening matrix is given by $\boldsymbol{\Lambda}_{\mathbf{n}}^{-\frac{1}{2}} \mathbf{E}_{\mathbf{n}}{ }^{H}$, where $\boldsymbol{\Lambda}_{n} \in \mathbb{C}^{2 \times 2}$ and $\mathbf{E}_{\mathbf{n}} \in \mathbb{C}^{2 \times 2}$ contain respectively the eigenvalues and eigenvectors of the covariance matrix $\mathbf{R}_{\nu_{\mathbf{n}}} \in \mathbb{C}^{2 \times 2}$ of the vector $\boldsymbol{\nu}_{n}=\left[\begin{array}{ll}\nu_{1 n} & \nu_{2 n}\end{array}\right]^{T} \in \mathbb{C}^{2}$.

The instantaneous SNR is then given by

$$
\gamma_{n}=P_{s} \mathbf{h}_{\mathbf{n}}^{\mathbf{H}} \mathbf{R}_{\nu}^{-\mathbf{1}} \mathbf{h}_{\mathbf{n}}
$$

where $\mathbf{h}_{\mathbf{n}}=\left[\begin{array}{ll}h_{1 n} & h_{2 n}\end{array}\right]^{T} \in \mathbb{C}^{2}$. By using (9)-(12), (13) can be rewritten as

$\gamma_{n}=\frac{P_{s}}{\operatorname{det} \mathbf{R}_{\nu_{\mathbf{n}}}}\left[h_{1 n}^{*} h_{2 n}^{*}\right]\left[\begin{array}{cc}\sigma_{\nu_{2 n}}^{2} & -\rho_{\nu_{1 n}, \nu_{2 n}} \\ -\rho_{\nu_{1 n}, \nu_{2 n}}^{*} & \sigma_{\nu_{1 n}}^{2}\end{array}\right]\left[\begin{array}{c}h_{1 n} \\ h_{2 n}\end{array}\right]$,

with $\operatorname{det} \mathbf{R}_{\nu_{\mathbf{n}}}=\sigma_{\nu_{1 n}}^{2} \sigma_{\nu_{2 n}}^{2}-\left|\rho_{\nu_{1 n}, \nu_{2 n}}\right|^{2}$,

$$
\begin{aligned}
\sigma_{\nu_{1 n}}^{2} & =\left|h_{n}^{(S R)}\right|^{2}\left|h_{n}^{(R D)}\right|^{2}\left|\alpha^{(R)}\right|^{2} g_{n}^{2} \sigma_{d^{(S)}}^{2} \\
& +\left|h_{n}^{(R D)}\right|^{2}\left|\alpha^{(R)}\right|^{2} g_{n}^{2} \sigma_{\eta}^{2}+\left|h_{n}^{(R D)}\right|^{2} \sigma_{d^{(R)}}^{2}+\sigma_{\eta}^{2}
\end{aligned}
$$

$\sigma_{\nu_{2 n}}^{2}=\left|h_{n}^{(S D)}\right|^{2} \sigma_{d^{(S)}}^{2}+\sigma_{\eta}^{2}, \quad$ and $\quad \rho_{\nu_{1 n}, \nu_{2 n}}=$ $h_{n}^{(S D)^{*}} h_{n}^{(S R)} h_{n}^{(R D)} g_{n} \alpha^{(R)} \sigma_{d^{(S)}}^{2}$, where $\sigma_{d^{(R)}}^{2}$ is the variance of $d_{n}^{(R)}$.

Let us define the following instantaneous SNRs of the wireless links:

$$
\begin{aligned}
\gamma_{n}^{(S D)} & =\left|h_{n}^{(S D)} \alpha^{(S)}\right|^{2} P_{s} / \sigma_{\eta}^{2}, \\
\gamma_{n}^{(S R)} & =\left|h_{n}^{(S R)} \alpha^{(S)}\right|^{2} P_{s} / \sigma_{\eta}^{2}, \\
\gamma_{n}^{(R D)} & =\left|h_{n}^{(R D)} \alpha^{(R)}\right|^{2} P_{r} / \sigma_{\eta}^{2},
\end{aligned}
$$

and the SNRs associated with the PAs:

$$
\begin{aligned}
& \gamma^{\left(P A_{S}\right)}=\left|\alpha^{(S)}\right|^{2} P_{s} / \sigma_{d^{(S)}}^{2}, \\
& \gamma^{\left(P A_{R}\right)}=\left|\alpha^{(R)}\right|^{2} P_{r} / \sigma_{d^{(R)}}^{2} .
\end{aligned}
$$

Note that $\gamma^{\left(P A_{S}\right)}$ (resp. $\gamma^{\left(P A_{R}\right)}$ ) corresponds to the SNR of the source's (resp. relay's) PA by considering only the effects of the NLD inserted by the PA and regarding the NLD as an AWGN component, as the NLD is a white complex Gaussian process uncorrelated with $s_{n}$. We assume that $\gamma^{\left(P A_{S}\right)}$ and $\gamma^{\left(P A_{R}\right)}$ are fixed, as the variations of the PA parameters are generally much slower than the wireless channel variations. 
By using (17)-(20) and the definitions $\sigma_{\nu_{1 n}}^{2}, \sigma_{\nu_{2 n}}^{2}$ and $\rho_{\nu_{1 n}, \nu_{2 n}}$, after some algebraic manipulations, (14) can be written as

$$
\gamma_{n}=\gamma_{n}^{N U M} / \gamma_{n}^{D E N}
$$

with $\gamma_{n}^{N U M}$ and $\gamma_{n}^{D E N}$ given by (22) and (23), shown at the top of the next page.

It is worth mentioning some particular cases of the exact instantaneous SNR given by (21):

- When both PAs are linear, i.e. $\gamma^{\left(P A_{S}\right)}, \gamma^{\left(P A_{R}\right)} \rightarrow \infty$, (21) equals the SNR of a linear two-hop AF system with MRC [1], and when the source's PA is linear, i.e. $\gamma^{\left(P A_{S}\right)} \rightarrow \infty$, (21) equals the SNR presented in [6], [7].

- When the SD link is not available, i.e. $\gamma_{n}^{(S D)}=0$, $\gamma_{n}$ is given by: $\gamma_{n}=\gamma_{n}^{(R D)} \gamma_{n}^{(S R)} \gamma^{\left(P A_{S}\right)^{2}} \gamma^{\left(P A_{R}\right)} / \gamma_{n}^{D E N}$. Moreover, in this case, when the SNRs have medium or high values (SNRs higher than $10 \mathrm{~dB}$ ), it can easily be shown that $\gamma_{n} \cong\left(\frac{1}{\gamma_{n}^{(S R)}}+\frac{1}{\gamma_{n}^{(R D)}}+\frac{1}{\gamma^{\left(P A_{S}\right)}}+\frac{1}{\gamma^{\left(P A_{R}\right)}}\right)^{-1}$, or

$$
\gamma_{n} \cong \min \left(\gamma_{n}^{(S R)}, \gamma^{\left(P A_{R}\right)}, \gamma^{\left(P A_{S}\right)}, \gamma_{n}^{(R D)}\right),
$$

which shows that SRD link can be viewed as a four-hop linear AF cooperative link, with individual SNRs given by $\gamma_{n}^{(S R)}, \gamma^{\left(P A_{R}\right)}, \gamma^{\left(P A_{S}\right)}$ and $\gamma_{n}^{(R D)}$ [10]. It is worth mentioning that (24) holds true when one of the SNRs is much smaller than the others. In several previous works, e.g. [10], expressions similar to (24) have been used as approximations for $\gamma_{n}$ in multihop relaying systems with good accuracy.

- When the relay is not available, i.e. $\gamma_{n}^{(S R)}=0$ or $\gamma_{n}^{(R D)}=$ 0, (21) becomes: $\gamma_{n}=\left[1 / \gamma_{n}^{(S D)}+1 / \gamma^{\left(P A_{S}\right)}\right]^{-1}$, or

$$
\gamma_{n} \cong \min \left(\gamma_{n}^{(S D)}, \gamma^{\left(P A_{S}\right)}\right),
$$

which shows that SD link can be viewed as a two-hop linear AF cooperative link, with individual SNRs given by $\gamma_{n}^{(S D)}$ and $\gamma^{\left(P A_{S}\right)}$ [6], [7], [10]. Similarly, (25) holds true when one of the SNRs is much smaller than the other one. In several previous works, expressions similar to (25) have been used as approximations for $\gamma_{n}$ in two-hop AF cooperative relaying systems with good accuracy [6], [7], [10].

\section{Outage Probability with MRC}

In this section, a closed form expression for an approximation of the outage probability is derived, for the MRC method and Rayleigh fading. In the sequel, $f_{X}(\cdot)$ and $F_{X}(\cdot)$ denote respectively the probability density function (PDF) and cumulative distribution function $(\mathrm{CDF})$ of the random variable $X$.

As the received signals $x_{n}^{(S R D)}$ and $x_{n}^{(S D)}$ have correlated noises, the covariance matrix $\mathbf{R}_{\nu_{\mathbf{n}}}$ of the noise vector $\boldsymbol{\nu}_{n}$ is not diagonal. However, for mild PA nonlinearity, the vector $\boldsymbol{\nu}_{n}$ exhibits generally a low correlation coefficient. Indeed, in our simulations with Rayleigh fading and softclipping PAs, the correlation coefficient associated with $\boldsymbol{\nu}_{n}$ has values typically below 0.25 . That allows us to make the following approximation: $\gamma_{n} \cong \gamma_{n}^{(S R D)}+\gamma_{n}^{(S D)}$, where $\gamma_{n}^{(S R D)}$ is the SNR when the SD link is not available and $\gamma_{n}^{(S D)}$ is the SNR when the relay node is not available.
Using (24) and (25), we may then write $\gamma_{n} \cong \gamma_{n}^{(\min 1)}+$ $\gamma_{n}^{(\min 2)}$, with $\gamma_{n}^{(\min 1)}=\min \left(\gamma_{n}^{(S D)}, \gamma^{\left(P A_{S}\right)}\right)$ and $\gamma_{n}^{(\min 2)}=$ $\min \left(\gamma_{n}^{(S R)}, \gamma^{\left(P A_{R}\right)}, \gamma^{\left(P A_{S}\right)}, \gamma_{n}^{(R D)}\right)$.

The approximated outage probability is then given by

$$
\begin{aligned}
P_{n}^{(\text {out }, m r c)}\left(\gamma_{t h}\right) & =P\left(\gamma_{n}^{(\min 1)}+\gamma_{n}^{(\min 2)}<\gamma_{t h}\right) \\
& =\int_{-\infty}^{+\infty} f_{\gamma_{n}^{(\min 1)}}(x) F_{\gamma_{n}^{(\min 2)}}\left(\gamma_{t h}-x\right) d x
\end{aligned}
$$

where $\gamma_{t h}$ is a SNR threshold, with the CDF of $\gamma_{n}^{(\min 2)}$ expressed as

$$
\begin{aligned}
F_{\gamma_{n}^{(m i n 2)}}\left(\gamma_{t h}\right)=1 & -\left[1-F_{\gamma_{n}^{(S R)}}\left(\gamma_{t h}\right)\right]\left[1-F_{\gamma^{\left(P A_{R}\right)}}\left(\gamma_{t h}\right)\right] \\
& \times\left[1-F_{\gamma_{n}^{(R D)}}\left(\gamma_{t h}\right)\right]\left[1-F_{\gamma^{\left(P A_{S}\right)}}\left(\gamma_{t h}\right)\right]
\end{aligned}
$$

Although $\gamma^{\left(P A_{S}\right)}$ and $\gamma^{\left(P A_{R}\right)}$ are fixed, we can associate CDFs to these SNRs, in the following way: $F_{\gamma^{\left(P A_{S}\right)}}\left(\gamma_{t h}\right)=$ $u\left(\gamma_{t h}-\bar{\gamma}^{\left(P A_{S}\right)}\right)$ and $F_{\gamma^{\left(P A_{R}\right)}}\left(\gamma_{t h}\right)=u\left(\gamma_{t h}-\bar{\gamma}^{\left(P A_{R}\right)}\right)$, where $u(\cdot)$ is the unit step function, $\gamma^{\left(P A_{S}\right)}=\bar{\gamma}^{\left(P A_{S}\right)}$ and $\gamma^{\left(P A_{R}\right)}=$ $\bar{\gamma}^{\left(P A_{R}\right)}$. Moreover, as Rayleigh fading is considered at the SR and RD links, $F_{\gamma_{n}^{(\min 2)}}\left(\gamma_{t h}\right)$ can be written as

$$
\begin{aligned}
F_{\gamma_{n}^{(\min 2)}}\left(\gamma_{t h}\right) & =u\left(\gamma_{t h}\right)-\exp \left[-\gamma_{t h}\left(\frac{1}{\bar{\gamma}^{(S R)}}+\frac{1}{\bar{\gamma}^{(R D)}}\right)\right] \\
& \times u\left(\gamma_{t h}\right) u\left(-\gamma_{t h}+\min \left(\bar{\gamma}^{\left(P A_{S}\right)}, \bar{\gamma}^{\left(P A_{R}\right)}\right)\right)
\end{aligned}
$$

where $\bar{\gamma}^{(S R)}$ and $\bar{\gamma}^{(R D)}$ are the mean values of $\gamma_{n}^{(S R)} \mathrm{e}$ $\gamma_{n}^{(R D)}$, respectively. Equation (28) corresponds to the outage probability when only the SRD is available. Similarly, we have

$$
\begin{aligned}
F_{\left.\gamma_{n}^{(m i n} 1\right)}\left(\gamma_{t h}\right) & =u\left(\gamma_{t h}\right)-\exp \left(-\frac{\gamma_{t h}}{\bar{\gamma}^{(S D)}}\right) \\
& \times u\left(\gamma_{t h}\right) u\left(\bar{\gamma}^{\left(P A_{S}\right)}-\gamma_{t h}\right),
\end{aligned}
$$

where $\bar{\gamma}^{(S D)}$ is mean of $\gamma_{n}^{(S D)}$. By differentiating $F_{\gamma_{n}^{(\min 1)}}\left(\gamma_{t h}\right)$, we get

$$
\begin{array}{r}
f_{\gamma_{n}^{(m i n)}}\left(\gamma_{t h}\right)=\frac{1}{\bar{\gamma}^{(S D)}} \exp \left(-\frac{\gamma_{t h}}{\bar{\gamma}^{(S D)}}\right) u\left(-\gamma_{t h}+\gamma^{\left(P A_{S}\right)}\right) \\
+\exp \left(-\frac{\gamma_{t h}}{\bar{\gamma}^{(S D)}}\right) \delta\left(-\gamma_{t h}+\gamma^{\left(P A_{S}\right)}\right)
\end{array}
$$

$F_{\gamma_{(\min 1)}}\left(\gamma_{t h}\right)$ corresponds to the outage probability when only the SD is available.

Substituting (28) and (30) into (26), and making some algebraic manipulations, we get (31), shown at the top of the page, where $\Psi=\max \left(\gamma_{t h}-\min \left(\bar{\gamma}^{\left(P A_{S}\right)}, \bar{\gamma}^{\left(P A_{R}\right)}\right), 0\right)$ and $\bar{\gamma}^{M}=\left(-\frac{1}{\bar{\gamma}^{(S D)}}+\frac{1}{\bar{\gamma}^{(S R)}}+\frac{1}{\bar{\gamma}^{(R D)}}\right)^{-1}$, assuming $-\frac{1}{\bar{\gamma}^{(S D)}}+$ $\frac{1}{\bar{\gamma}^{(S R)}}+\frac{1}{\bar{\gamma}^{(R D)}} \neq 0$.

In order to have a better understanding of (31), Table 1 shows the expressions of $P_{n}^{(o u t, m r c)}\left(\gamma_{t h}\right)$ in four different cases, depending on the value of $\gamma_{t h}$, with $\gamma^{\left(P A_{\min }\right)}=$ $\min \left(\bar{\gamma}^{\left(P A_{S}\right)}, \bar{\gamma}^{\left(P A_{R}\right)}\right)$. It can be demonstrated that the Line 1 of Table 1 corresponds to an order of diversity equal to 2, while Lines 2 and 3 correspond to an order of diversity equal to 1 , and Line 4 represents an order of diversity equal to zero. The demonstrations are omitted due to lack of space. 


$$
\begin{gathered}
\gamma_{n}^{N U M}=\gamma_{n}^{(R D)} \gamma_{n}^{(S R)} \gamma_{n}^{\left(P A_{S}\right)^{2}} \gamma^{\left(P A_{R}\right)}+\gamma_{n}^{(R D)} \gamma_{n}^{(S D)} \gamma_{n}^{\left(P A_{S}\right)^{2}} \gamma^{\left(P A_{R}\right)} \\
+\gamma_{n}^{(S D)} \gamma_{n}^{(R D)} \gamma^{\left(P A_{S}\right)}\left[\gamma_{n}^{(S R)} \gamma^{\left(P A_{S}\right)}+\gamma_{n}^{(S R)}+\gamma^{\left(P A_{S}\right)}+\gamma_{n}^{(S D)} \gamma^{\left(P A_{S}\right)} \gamma^{\left(P A_{R}\right)}\left[\gamma_{n}^{(S R)} \gamma^{\left(P A_{S}\right)}+\gamma_{n}^{(S R)}+\gamma^{\left(P A_{S}\right)}\right]\right. \\
\gamma_{n}^{D E N}=\gamma_{n}^{(R D)} \gamma_{n}^{(S D)} \gamma^{\left(P A_{R}\right)} \gamma^{\left(P A_{S}\right)}+\gamma_{n}^{(R D)} \gamma_{n}^{(S D)}\left[\gamma_{n}^{(S R)} \gamma^{\left(P A_{S}\right)}+\gamma_{n}^{(S R)}+\gamma^{\left(P A_{S}\right)}\right]+\gamma_{n}^{(R D)} \gamma_{n}^{\left(P A_{S}\right)^{2}} \gamma^{\left(P A_{R}\right)} \\
+\gamma_{n}^{(S D)} \gamma^{\left(P A_{R}\right)}\left[\gamma_{n}^{(S R)} \gamma^{\left(P A_{S}\right)}+\gamma_{n}^{(S R)}+\gamma^{\left(P A_{S}\right)}\right]+\gamma_{n}^{(R D)} \gamma^{\left(P A_{S}\right)}\left[\gamma_{n}^{(S R)} \gamma^{\left(P A_{S}\right)}+\gamma_{n}^{(S R)}+\gamma^{\left(P A_{S}\right)}\right] \\
+\gamma^{\left(P A_{R}\right)} \gamma^{\left(P A_{S}\right)}\left[\gamma_{n}^{(S R)} \gamma^{\left(P A_{S}\right)}+\gamma_{n}^{(S R)}+\gamma^{\left(P A_{S}\right)}+\gamma_{n}^{(S R)} \gamma_{n}^{(R D)} \gamma^{\left(P A_{R}\right)} \gamma^{\left(P A_{S}\right)}\right. \\
P_{n}^{(o u t, m r c)}\left(\gamma_{t h}\right)=1-\exp \left(-\frac{\gamma_{t h}}{\left.\bar{\gamma}^{(S D)}\right)}\right) u\left(\gamma^{\left(P A_{S}\right)}-\gamma_{t h}\right)-\exp \left(-\frac{\gamma_{t h}}{\bar{\gamma}^{(S R)}}-\frac{\gamma_{t h}}{\left.\bar{\gamma}^{(R D)}\right)}\right) \\
\times\left[\int_{\Psi}^{\gamma_{t h}} \frac{1}{\bar{\gamma}^{(S D)}} \exp \left(\frac{x}{\bar{\gamma}^{M}}\right) u\left(-x+\gamma^{\left(P A_{S}\right)}\right) d x+\int_{\Psi}^{\gamma_{t h}} \exp \left(\frac{x}{\bar{\gamma}^{M}}\right) \delta\left(-x+\gamma^{\left(P A_{S}\right)}\right) d x\right]
\end{gathered}
$$

Besides, the expression for $P_{n}^{(o u t, m r c)}\left(\gamma_{t h}\right)$ in Line 1 is the same as in the case where both amplifiers are linear and Line 2 corresponds to the case where only the relay PA is nonlinear [6], [7].

\section{Outage Probability with SC}

In this section, a closed form expression for an approximation of the outage probability is derived considering the SC receiver. In this case, we have: $\gamma_{n}=\max \left(\gamma_{n}^{(S R D)}, \gamma_{n}^{(S D)}\right)$. Assuming that $x_{n}^{(S R D)}$ and $x_{n}^{(S D)}$ are weakly correlated, it follows that: $P_{n}^{(\text {out }, s c)}\left(\gamma_{t h}\right)\left(\gamma_{t h}\right) \cong F_{\gamma_{n}^{(S R D)}}\left(\gamma_{t h}\right) F_{\gamma_{n}^{(S D)}}\left(\gamma_{t h}\right)$ and, hence

$$
P_{n}^{(\text {out }, s c)}\left(\gamma_{t h}\right) \cong F_{\gamma_{n}^{(m i n 1)}}\left(\gamma_{t h}\right) F_{\gamma_{n}^{(m i n 2)}}\left(\gamma_{t h}\right)
$$

with $F_{\gamma_{n}^{(\min 1)}}\left(\gamma_{t h}\right)$ and $F_{\gamma_{n}^{(\min 2)}}\left(\gamma_{t h}\right)$ given in Section IV. That leads to the expressions in Table 2, which shows the analytical expressions of $P_{n}^{(o u t, s c)}\left(\gamma_{t h}\right)$ in three different cases, depending on the value of $\gamma_{t h}$, for the SC receiver.

In this case, it can be shown that Line 1 corresponds to an order of diversity equal to 2 , Line 2 to an order of diversity of 1 and Line 3 to an order of diversity equal to zero. The demonstrations are omitted due to lack of space. The expression for $P_{n}^{(o u t, s c)}\left(\gamma_{t h}\right)$ in Line 1 is the same as in the case where both amplifiers are linear [7].

\section{Discussion}

The results derived in Sections IV and V show that, for both the MRC and SC receivers, the nonlinearities of the PAs do not affect the outage probability and the diversity order for $\gamma^{\left(P A_{\text {min }}\right)}>\gamma_{t h}$, i.e. when the SNR threshold $\gamma_{t h}$ is small and/or when $\bar{\gamma}^{\left(P A_{S}\right)}$ and $\bar{\gamma}^{\left(P A_{R}\right)}$ are high. This behavior can be explained by regarding the SRD link as a series-cascade of four linear channels, two of them being the fixed PA models. As the SNR $\gamma_{n}^{(\min 2)}$ of the global SRD link is limited by the worst of the four links, when $\gamma_{t h}$ is smaller than $\bar{\gamma}^{\left(P A_{S}\right)}$ or $\bar{\gamma}^{\left(P A_{R}\right)}$, the SRD link will never be in outage due to the presence of the PAs, that is, $\bar{\gamma}^{\left(P A_{S}\right)}$ and $\bar{\gamma}^{\left(P A_{R}\right)}$ will have no impact on the outage probability. A similar reasoning can be derived for the SD link, which means that both the SRD and SD links act as linear links in this situation. This explains why $P_{n}^{(\text {out }, m r c)}\left(\gamma_{t h}\right)$ in Line 1 of Tables I and II are the same as in the case where both amplifiers are linear. It is important to mention that a value of $\bar{\gamma}^{\left(P A_{S}\right)}$ (or $\left.\bar{\gamma}^{\left(P A_{R}\right)}\right)$ occurs when the PA has a high saturation power and/or when the transmission power is small.

On the other hand, when $\gamma_{t h}$ is too high $\left(\gamma_{t h}>\gamma^{\left(P A_{S}\right)}+\right.$ $\left.\gamma^{\left(P A_{\min }\right)}\right), \gamma_{n}^{(\min 1)}$ and $\gamma_{n}^{(\min 2)}$ are limited by the SNRs of the PAs, regardless of the quality of the wireless channels. This means that both the SRD and SD paths are always in outage. This explains why $P_{n}^{(o u t, m r c)}\left(\gamma_{t h}\right)=1$ in Line 4 of Table I and in Line 3 of Table II.

When $\bar{\gamma}^{\left(P A_{S}\right)}>\gamma_{t h}>\bar{\gamma}^{\left(P A_{R}\right)}$, the diversity gain provided by the relay is lost due to the NLD inserted by the PA of the relay, but the SD link won't be affected by the PA. In this case, the SRD link is always in outage, but the SD link acts as a linear link. This explains why Line 2 of Table I corresponds to the case where only the relay's PA is nonlinear and the source's PA is linear.

Note also that, with the SC receiver, the system is always in outage when $\gamma_{t h}>\gamma^{\left(P A_{S}\right)}$, regardless of the value of $\bar{\gamma}^{\left(P A_{S}\right)}$, which does not happen with the MRC receiver (Line 3 of Table I). This is due to the fact that the SNR of the MRC receiver is the sum of the SNRs of the SRD and SD links. Thus, even if both links are in outage, it is possible that their sum is not in outage, which is not the case for the SC receiver.

These results show that the PA of the source has a higher impact on the outage probability than the PA of the relay. Indeed, when $\gamma_{t h}>2 \bar{\gamma}^{\left(P A_{S}\right)}$, the system is always in outage, for both the MRC and SC receivers.

\section{Simulation Results}

In this section, the theoretical expressions derived in this paper are evaluated by means of computer simulations, with $N=256$ subcarriers, 16-QAM signals, $10^{5}$ Monte Carlo runs and PAs modeled by the soft-clipping (soft limiter) model. Closed-form expressions for $\alpha^{(S)}, \alpha^{(R)}, \sigma_{d^{(S)}}^{2}$ and $\sigma_{d^{(R)}}^{2}$ are given in [3]. It is also assumed that $\bar{\gamma}^{(S D)}=\bar{\gamma}^{(S R)}=\bar{\gamma}^{(R D)}=$ $\bar{\gamma}$ and that perfect channel state information is known at the receivers. Moreover, we used $P_{s}=0.31$ and $P_{r}=1$, which leads to $\bar{\gamma}^{\left(P A_{S}\right)}=23.5 \mathrm{~dB}$ and $\bar{\gamma}^{\left(P A_{R}\right)}=12.0 \mathrm{~dB}$.

Figs. 1 and 2 show the outage probability versus the mean SNR $\bar{\gamma}$ for the MRC and SC receivers, respectively, with 
TABLE I: Outage Probability with MRC

\begin{tabular}{|c|c|c|c|c|}
\hline Range of $\gamma_{t h}$ & \multicolumn{4}{|c|}{ Expression for $P_{n}^{(o u t, m r c)}\left(\gamma_{t h}\right)$} \\
\hline$\gamma^{\left(P A_{m i n}\right)}>\gamma_{t h}$ & \multicolumn{4}{|c|}{ 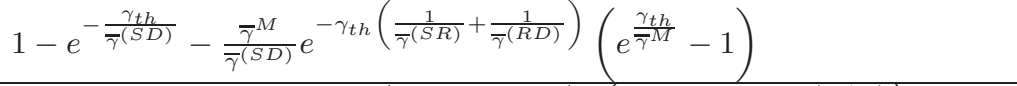 } \\
\hline $\bar{\gamma}^{\left(P A_{S}\right)}>\gamma_{t h}>\bar{\gamma}^{\left(P A_{R}\right)}$ & \multicolumn{4}{|c|}{ 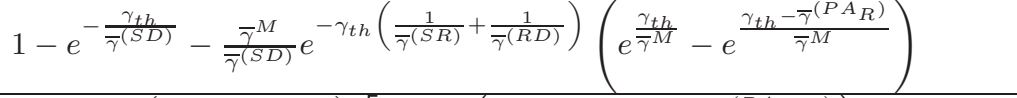 } \\
\hline$\gamma^{\left(P A_{S}\right)}+\gamma^{\left(P A_{m i n}\right)}>\gamma_{t h}>\gamma^{\left(P A_{S}\right)}$ & $1-e^{-\gamma_{t h}\left(\frac{1}{\bar{\gamma}^{(S R)}}+\frac{1}{\bar{\gamma}^{(R D)}}\right)}$ & $\frac{\bar{\gamma}^{M}}{\bar{\gamma}^{(S D)}}\left(e^{\frac{\gamma^{\left(P A_{S}\right)}}{\bar{\gamma}^{M}}}\right.$ & $-e^{\frac{\gamma_{t h}-\gamma^{\left(P A_{\min }\right)}}{\bar{\gamma}^{M}}}$ & $+e^{\frac{\gamma^{\left(P A_{S}\right)}}{\bar{\gamma}^{M}}}$ \\
\hline$\gamma_{t h}>\gamma^{\left(P A_{S}\right)}+\gamma^{\left(P A_{\min }\right)}$ & 1 & & & \\
\hline
\end{tabular}

TABLE II: Outage Probability with SC

\begin{tabular}{|l|l|}
\hline Range of $\gamma_{t h}$ & \multicolumn{2}{|l|}{ Expression for $P_{n}^{(o u t, s c)}\left(\gamma_{t h}\right)$} \\
\hline$\gamma^{\left(P A_{\min }\right)}>\gamma_{t h}$ & {$\left[1-\exp \left(-\frac{\gamma_{t h}}{\bar{\gamma}^{(S D)}}\right)\right]\left[1-\exp \left[-\gamma_{t h}\left(\frac{1}{\bar{\gamma}^{(S R)}}+\frac{1}{\bar{\gamma}^{(R D)}}\right)\right]\right]$} \\
\hline $\bar{\gamma}^{\left(P A_{S}\right)}>\gamma_{t h}>\bar{\gamma}^{\left(P A_{R}\right)}$ & $1-\exp \left(-\frac{\gamma_{t h}}{\bar{\gamma}^{(S D)}}\right)$ \\
\hline$\gamma_{t h}>\gamma^{\left(P A_{S}\right)}$ & 1 \\
\hline
\end{tabular}

various values of $\gamma_{t h}$. The following outage probability curves are shown: simulated with nonlinear PAs, simulated with linear PAs and theoretical with nonlinear PAs. From these figures, it can be noticed that the proposed expressions are close to the simulated curves for all the tested cases. Moreover, from the slopes of the curves, it can be inferred that the diversity orders predicted in Sections IV and V are correct.

By comparing the curves obtained with linear PAs with the ones obtained with nonlinear PAs, it can be deduced that the outage probability is significantly affected by the nonlinearities only if $\gamma^{\left(P A_{\min }\right)}=12.0 \mathrm{~dB}<\gamma_{t h}$, which means that the nonlinear PA considerably increases the outage probability and decreases the system diversity only for high SNR thresholds $\gamma_{t h}$. As mentioned earlier, this is due to the fact that, as the global SRD link is limited by the worst of four SNRs, when $\gamma_{t h}$ is too high, the SRD link performance is limited by $\gamma^{\left(P A_{m i n}\right)}$, which means that the SRD path is unable to provide diversity. A similar reasoning can be derived for the SD link.

In addition, by comparing Figs. 1 and 2, it can be viewed that the outage probabilities provided by the MRC are slightly smaller than the ones obtained with the SC receiver, for the most part of the tested cases. The exception to this behavior occurs for $\gamma_{t h}=25 \mathrm{~dB}$ and $\gamma_{t h}=30 \mathrm{~dB}$, which leads to an outage probability equal to one in both cases.

Fig. 3 shows the theoretical outage probability versus $\gamma_{t h}$ for a fixed SNR of $25 d B$, obtained with the MRC and SC receivers. It can be confirmed from this figure that, when $\gamma^{\left(P A_{\text {min }}\right)}>\gamma_{t h}$, the nonlinear PAs have a small impact on the outage probability. For $\bar{\gamma}^{\left(P A_{R}\right)}<\gamma_{t h}$, the PA nonlinearities have a higher impact on the system performance. Moreover, as expected, when $\bar{\gamma}^{\left(P A_{S}\right)}<\gamma_{t h}$, we have $P_{n}^{(\text {out }, m r c)}\left(\gamma_{t h}\right)=$ $P_{n}^{(\text {out }, s c)}\left(\gamma_{t h}\right)=1$. This figure also confirms that the outage probabilities of the MRC are slightly smaller than the ones of the SC receiver, for the most part of the tested cases.

\section{CONCLUSION}

It can be concluded that the proposed expressions are very close to the simulated curves and that the outage probability is significantly increased only for $\gamma^{\left(P A_{\min }\right)}<\gamma_{t h}$. In fact, the diversity order of the system depends on the values of

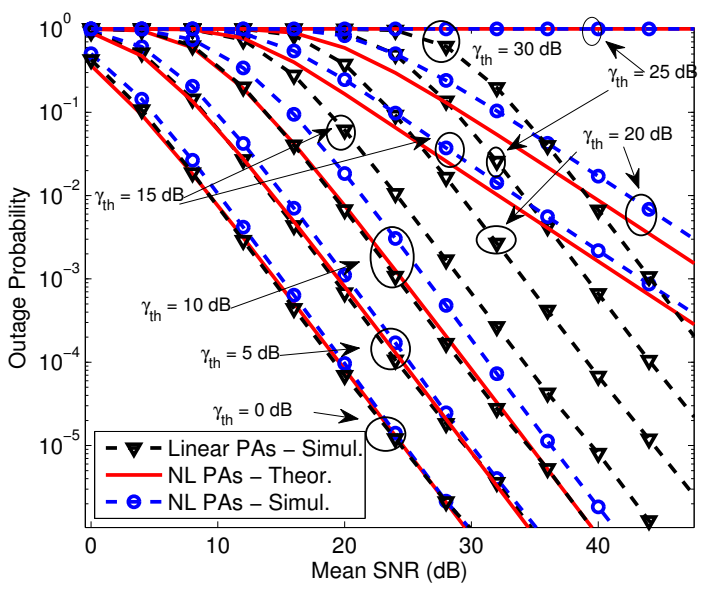

Fig. 1: Outage probability versus the mean SNR for the MRC receiver.

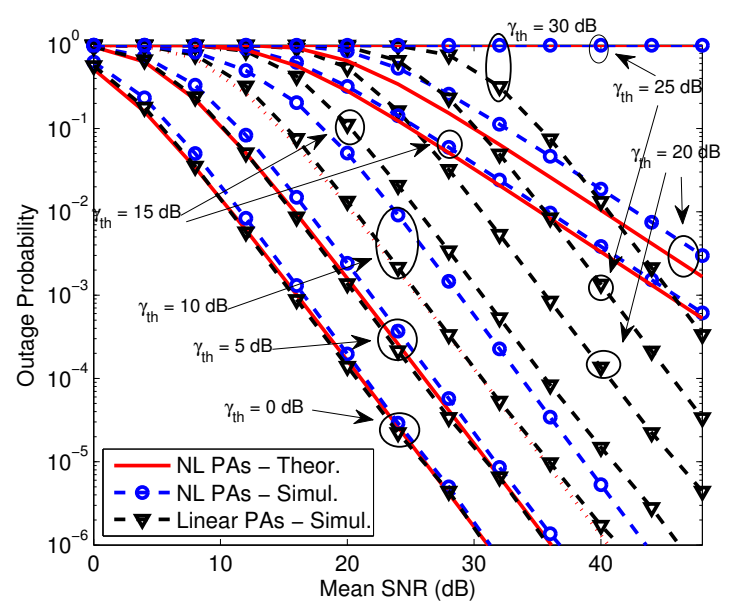

Fig. 2: Outage probability versus the mean SNR for the SC receiver.

$\gamma^{\left(P A_{S}\right)}, \gamma^{\left(P A_{R}\right)}$ and $\gamma_{t h}$. Moreover, it can also be deduced that the MRC and SC techniques provide roughly the same outage probabilities, except when $\gamma^{\left(P A_{S}\right)}+\gamma^{\left(P A_{m i n}\right)}>\gamma_{t h}>$ $\gamma^{\left(P A_{S}\right)}$. In future works, this analysis will be extended to more general fading and system models, and a SER analysis will be developed. 


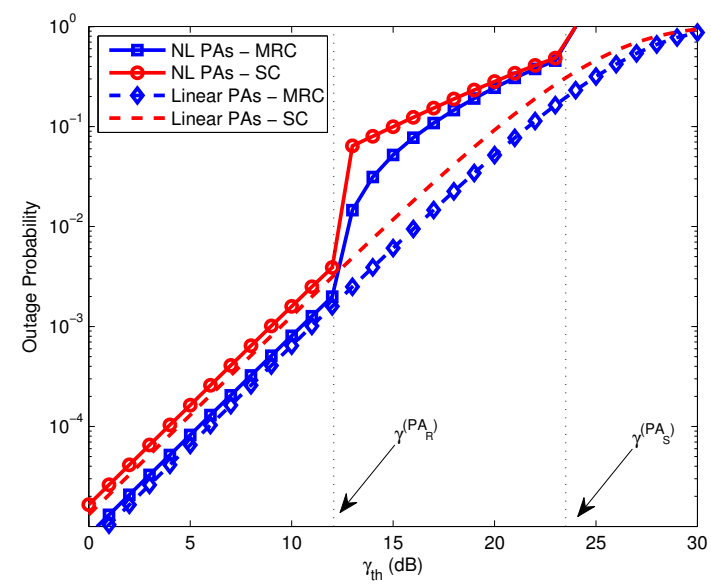

Fig. 3: Outage probability versus $\gamma_{t h}$ for the MRC and SC receivers.

\section{REFERENCES}

[1] J. N. Laneman, D. N. C. Tse, and G. W. Wornell, "Cooperative diversity in wireless networks: efficient protocols and outage behavior," IEEE Transactions on Information Theory, vol. 50, no. 12, pp. 3062-3080, Dec. 2004. doi: 10.1109/TIT.2004.838089.

[2] G. W. S. L. T. Hwang, C. Yang and G. Y. Li, "OFDM and its wireless applications: a survey," IEEE Transactions on Vehicular Technology, vol. 58, no. 4, pp. 1673-1694, May 2009. doi: 10.1109/TVT.2008.2004555.

[3] D. Dardari, V. Tralli, and A. Vaccari, "A theoretical characterization of nonlinear distortion effects in OFDM systems," IEEE Transactions on Communications, vol. 48, no. 10, pp. 1755-1764, Oct. 2000. doi: $10.1109 / 26.871400$.

[4] T. Riihonen, S. Werner, F. Gregorio, R. Wichman, and J. Hamalainen, "BEP analysis of OFDM relay links with nonlinear power amplifiers," in IEEE Wireless Communications and Networking Conference (WCNC), Sydney, Australia, Apr. 2010, pp. 1-6. doi: 10.1109/WCNC.2010.5 506301

[5] H. A. Ahmed, A. I. Sulyman, and H. S. Hassanein, "Bit error rate performance of orthogonal frequency-division multiplexing relaying systems with high power amplifiers and Doppler effects," Wireless Communications and Mobile Computing, Apr. 2011. doi: 10.1002/wcm.1135.

[6] C. A. R. Fernandes, "Outage performance of cooperative amplifyand-forward OFDM systems with nonlinear power amplifiers," in IEEE International Workshop Signal Processing Advances in Wireless Communications (SPAWC), Cesme, Turkey, Jun. 2012. doi: 10.1109/SPAWC.2012.6292949.

[7] C. A. R. Fernandes and D. B. da Costa and A. L. F. de Almeida, "Performance analysis of cooperative amplify-and-forward OFDM systems with power amplifier nonlinearity," IET Communications, vol. 8, no. 18, pp. 3223-3233, Dec. 2014. doi: 10.1049/iet-com.2014.0369.

[8] M. M. Eddaghel, U. N. Mannai and J. A. Chambers, "Outage Probability Analysis of an AF Cooperative Multi-Relay Network with Best Relay Selection and Clipped OFDM Transmission," in International Symposium on Wireless Communication Systems, Ilmenau, Germany, August 2013, pp. 1-5.

[9] C. A. R. F. E. J. P. Farias, D. A. Sousa and C. E. R. Fernandes, "Receptor Ótimo para sistemas OFDM cooperativos com amplificadores de potência não lineares," in Brazilian Telecommunications Symposium, Brazilia, Brazil, Sep. 2012.

[10] M. O. Hasna and M.-S. Alouini, "Outage probability of multihop transmission over nakagami fading channels," IEEE Coтmunications Letters, vol. 5, no. 7, pp. 1089-7798, May 2003. doi: 10.1109/LCOMM.2003.812178

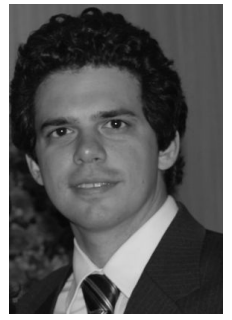

C. Alexandre R. Fernandes C. Alexandre R. Fernandes was born in Fortaleza, Brazil, in 1981. He received the B.Sc. degree in Electrical Engineering from the Universidade Federal do Ceará (UFC), Brazil, in 2003, M.Sc. degrees from the UFC/Brazil and University of Nice Sophia-Antipolis (UNSA), France, in 2005, and the double Ph.D. degree from the UFC/Brazil and UNSA/France, in 2009, in telecommunications engineering.

In 2008 and 2009, he was Teaching Assistant with the UNSA/France and, from Jul/2009 to Feb/2010, he was a Postdoctoral Fellow with the Department of Teleinformatics Engineering of UFC/Brazil. In 2010, he joined the UFC/Brazil, where he now works as Assistant Professor with the Department of Computer Engineering in Sobral, being currently the Head of the graduate program in Electrical and Computer Engineering.

His research interest lies in the area of signal processing for wireless communications, and includes channel estimation and equalization, multicarrier systems, cooperative communications, tensor decompositions and nonlinear systems.

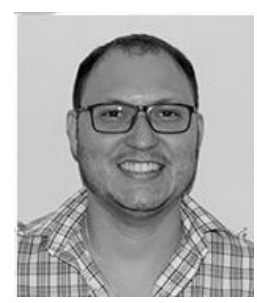

Éder J. P. Farias Eder J. P. Farias received the B.Sc. degree in Computer Science from the Universidade Estadual Vale do Acaraú (UVA), Brazil, in 2007, and the M.Sc. degree from the Universidade Federal do Ceará (UFC), Brazil, in 2013, in telecommunications engineering.

He is currently Professor and Managing Director with the Instituto Superior de Teologia Aplicada (INTA), Brazil, and Professor with the UVA/Brazil. His research interest lies in the area of signal processing for communications, and includes multicarrier systems, cooperative communications, nonlinear systems and network management. 